\title{
TWO-PERSON RED-AND-BLACK WITH BET-DEPENDENT WIN PROBABILITIES
}

\author{
LAURA PONTIGGIA, ${ }^{*}$ University of Minnesota
}

\begin{abstract}
We present two variations of a two-person, noncooperative stochastic game, inspired by the famous red-and-black gambling problem presented by Dubins and Savage. Two players each hold an integer amount of money and they each aim to win the other player's fortune. At every stage of the game they simultaneously bid an integer portion of their current fortune, and their probabilities of winning depend on these bids. We describe two different laws of motion specifying this dependency. In one version of the game, the players' probabilities of winning are proportional to their bets. In the other version, the probabilities of winning depend on the size of their bets and a weight parameter $w$. For each version we give a Nash equilibrium, in which the player for which the game is subfair $\left(w \leq \frac{1}{2}\right)$ plays boldly and the player for which the game is superfair $\left(w \geq \frac{1}{2}\right)$ plays timidly.
\end{abstract}

Keywords: Stochastic game; red-and-black; Nash equilibrium

2000 Mathematics Subject Classification: Primary 91A15

Secondary 91A05

\section{Introduction}

One of the most interesting examples in Dubins and Savage [3] is the famous red-and-black gambling problem. In the discrete version of this gambling problem, a player starts with an initial nonnegative integer fortune $x$ and wants to reach a goal $g$ by betting, at each stage of the game, an integer amount $s$ not greater than his current fortune. If his initial stake is $s \leq x$, with probability $w$ (with $0<w<1$ ) the player wins, and his fortune will be $x+s$ when he moves to the next stage of the game. With probability $\bar{w}=1-w$, the player loses and his fortune is decreased by $s$ before he moves to the next stage. And so on until the goal $g$ is reached or the player goes broke.

The problem for the player is to decide how many units of his current fortune should be staked at each stage in order to maximize his probability of reaching the goal $g$.

Dubins and Savage [3] showed that the solution of this problem depends on the value of the probability $w$. In particular, they showed that in the subfair case, when $w \leq \frac{1}{2}$, an optimal strategy is bold play, which corresponds to always staking the entire fortune or just what is needed to reach the target - whichever is smaller. In the superfair case (i.e. $w \geq \frac{1}{2}$ ) Ross [7] proved that it is optimal for the player to play timidly, that is, always to stake 1 unit of his current fortune when he is between 1 and $g-1$, and 0 otherwise. A gambling-theoretical proof of Ross's result can be found in Maitra and Sudderth [4].

Received 12 May 2004; revision received 29 September 2004.

* Postal address: School of Statistics, University of Minnesota, 313 Ford Hall, 224 Church Street SE, Minneapolis, MN 55455-0493, USA. Email address: pontla@stat.umn.edu 
Dubins [2] has considered a finite-fortune red-and-black game with a goal, in which the win probability depends on the fortune held by the player. He found a necessary and sufficient condition for timid play to be uniquely optimal.

Secchi [8] introduced the idea of a two-person red-and-black game as one in which player $I$ has an initial fortune $x$ and wants to reach a goal $g$, while player $I I$ wants to keep player $I$ from reaching his goal. He provides $\varepsilon$-optimal strategies for the players. An extension of red-and-black to $k$-player models is given in Pendergrass and Siegrist [5].

In this paper, we present two different formulations of a two-person red-and-black stochastic game, in which the probabilities of winning are not fixed, but can change at each stage of the game. In particular, we assume that they depend on the bets of the two players.

In these games, the transition probabilities are controlled by the players through their bets and by chance. This makes our games suitable for modeling situations where, for instance, there are two speculators who are willing to engage part of their fortunes in a risky business with the aim of reaching a goal that is represented by ownership of the entire stock. At each stage of the game, they each own an integer part of the entire stock and they have to decide which portion of their current stock they want to stake, knowing that the probabilities of winning depend on the size of the bets.

In Section 2, we introduce the notation and the terminology we will be using in the following sections, and set up the formulation of the two-person red-and-black stochastic games studied here. In Section 3, we examine a particular formulation of the game, called a weighted twoperson red-and-black game, in which the probability of a player winning at each stage of the game is defined as the product of a weight and the ratio of his bet and the sum of the two bets.

Section 4 is dedicated to a second formulation of the game, called proportional two-person red-and-black. In this game, we assign a weight to the bet made by each player and we define the probability of a player winning at each stage of the game as the ratio of his weighted bet and the sum of the two weighted bets.

We show that there are three possible situations for our two-person red-and-black games, which, by analogy with gambling theory, we call the subfair, the fair, and the superfair cases. As in a red-and-black gambling problem, optimal strategies for a player are different according to whether the game is subfair or superfair to him. In fact, for both formulations of the game we give a Nash equilibrium, in which the player for whom the game is subfair plays a bold strategy and the player for whom the game is superfair plays a timid strategy.

We will prove these results using a powerful technique that received an excellent treatment in Maitra and Sudderth [4], and was originally introduced by Dubins and Savage [3]. Our general procedure will be to guess a strategy for each player, find the function that gives the probability of reaching the goal with these strategies, and show that this function is excessive for one of the players, given the strategy of the other.

In the last section, we propose an extension of the proportional red-and-black game to an $N$-person model. When the game is not fair to all the players, we can only make a conjecture about a plausible Nash equilibrium.

\section{Preliminaries}

We imagine that there are two players who are engaged in a game, played in stages, where player $I$ has an initial, positive integer fortune of $x$ units and wants to reach a goal fortune of $g$ units by betting at each stage an amount not greater than his current fortune. However, player II also has an initial, positive integer fortune, and by staking at each stage an integer amount of units not greater than his current fortune, he wants to reach the same goal $g$. 
In particular, we assume that both players want to reach the target $g=M$, that is, the total amount of money in the system. We assume that $M$ is the same at each stage of the game. Therefore, if player $I$ starts with an initial positive fortune of $x$ units of money, then player $I I$ must have $M-x$ units of money. The game at each stage is zero sum: whatever amount one player wins, the other loses.

The state space of this game is represented by the set of possible fortunes held by player $I$, namely $S=\{0,1, \ldots, M\}$. The state space is discrete and the states 0 and $M$ are absorbing. In fact, after one of the players reaches his goal the game cannot move to any other state. In particular, only one of the players can reach the goal $M$.

At each stage, each player bids an amount of money that must be less than or equal to the fortune that he holds. We assume that each player chooses his action without any knowledge of the action chosen by the other player. In fact, all the games we consider are noncooperative in this sense.

Suppose that player $I$ bids an amount of money $a$ and player $I I$ bids an amount $b$, where $a \in A(x)=\{1, \ldots, x\}$ and $b \in B(x)=\{1, \ldots, M-x\}$ for $x=1, \ldots, M-1$, and $a=b=0$ if $x=0$ or $M$. In our games, we require players $I$ and $I I$ to bid at least 1 unit of money if they have a positive fortune less than $M$. If we allow them to bid 0 , then for these games there is a trivial, uninteresting Nash equilibrium, in which both players bid 0 forever, and the game stays in the same state perpetually.

The payoff function for each player is given by an indicator function, which takes value 1 if the player reaches his goal, and 0 otherwise. In all the games we consider, one player reaches the goal and the other reaches zero with probability 1 , so the games are almost surely constant sum.

Remark 2.1. Following the original formulation of the red-and-black gambling problem, we can assume that player $I$ wins the bet, moving to $x+b$, with some fixed probability $w$, and loses the bet, thereby moving to $x-a$, with probability $\bar{w}=1-w$. Notice that if the player has a positive fortune then he has a positive probability of losing at least 1 unit at each stage of the game.

Suppose that, at stage $m$, player $I$ has $1 \leq x^{m} \leq M-1$ units of money and he bids an amount $1 \leq a \leq x^{m}$ while player $I I$ bids $1 \leq b \leq M-x^{m}$. The law of motion for player $I$ at stage $m$ is defined by

$$
x^{m+1}= \begin{cases}x^{m}+b & \text { with probability } w \\ x^{m}-a & \text { with probability } \bar{w}=1-w .\end{cases}
$$

This version of a two-person red-and-black is very simple and it is trivial to prove that for any value of $w$ a Nash equilibrium is for both players to play timidly, which corresponds to always staking 1 unit of their current fortune at each stage of the game.

It seems likely that this is the unique nontrivial Nash equilibrium for this game. If bets of 0 were allowed, there would be a Nash equilibrium in which both players bid 0 forever. Notice also that in this game the trivial profile is not Pareto-optimal, but the timid profile is.

The primary purpose of this paper is to introduce formulations of this game in which the probabilities of winning are not fixed. In particular, we assume that after the players make their bets they will get back the total amount of money they staked with some probability that is proportional to how much they have bid. We also redefine a bold strategy as the strategy that corresponds to always staking the entire fortune. 


\section{Weighted two-person red-and-black}

We consider a two-person red-and-black game, in which each player's probability of winning depends on a weight $w$, and also on the size of the bets.

Suppose that, at stage $m$, player $I$ has $1 \leq x^{m} \leq M-1$ units of money and he bids an amount $1 \leq a \leq x^{m}$, and player $I I$ then has $1 \leq M-x^{m} \leq M-1$ units of money and bids $1 \leq b \leq M-x^{m}$. The law of motion for player $I$ at stage $m$ of the game is defined by

$$
x^{m+1}= \begin{cases}x^{m}+b & \text { with probability } \frac{a}{a+b} w \\ x^{m}-a & \text { with probability } 1-\frac{a}{a+b} w\end{cases}
$$

where the weight $w$ can take any value between 0 and 1 .

Notice that, for every pair of strategies for the two players, the process $\left\{x^{m}\right\}$ of the fortunes of player $I$ is a supermartingale. We say that the game is fair to both players if the process $\left\{x^{m}\right\}$ is a martingale. That means that if $x^{m}$ is player $I$ 's fortune at stage $m$ of the game, then his expected fortune at the next stage $m+1$ is equal to $x^{m}$ for all $m=0,1, \ldots$ In other words, the player's fortune on the next play is, on average, equal to his current fortune and is not otherwise affected by the previous history.

A weighted two-person red-and-black game will be a fair game if $w=1$.

Theorem 3.1. In a fair, weighted two-person red-and-black game, any pair of strategies with positive bets is Nash, and produces, for player $I$, the expected return $V_{1}(x)=x / M$ and, for player II, the expected return $V_{2}(x)=(M-x) / M$.

Proof. First we show that, for $w=1$, the process of fortunes of player $I$ is a martingale; i.e. that this is a fair game.

The law of motion for player $I$ at stage $m$ of the game is given by

$$
x^{m+1}= \begin{cases}x^{m}+b & \text { with probability } \frac{a}{a+b}, \\ x^{m}-a & \text { with probability } \frac{b}{a+b} .\end{cases}
$$

Therefore, taking the expectation of his fortune at stage $m+1$, we obtain

$$
\begin{aligned}
\mathrm{E}\left[x^{m+1} \mid x^{m}\right] & =\left(x^{m}+b\right) \frac{a}{a+b}+\left(x^{m}-a\right) \frac{b}{a+b} \\
& =x^{m} \frac{a}{a+b}+\frac{a b}{a+b}+x^{m} \frac{b}{a+b}-\frac{a b}{a+b} \\
& =x^{m} .
\end{aligned}
$$

We fix a strategy for player $I$ and a strategy for player $I I$, so that at stage $m$ player $I$ bids an integer amount $a$ and player $I I$ bids an integer amount $b$. Given this strategy profile, the expected return to player $I$, starting at $x$, is defined as

$$
V_{1}(x)=\mathrm{P}_{x}[\text { player } I \text { reaches } M]
$$


Therefore, we have the basic equation

$$
V_{1}\left(x^{m}\right)=\frac{a}{a+b} V_{1}\left(x^{m}+b\right)+\frac{b}{a+b} V_{1}\left(x^{m}-a\right),
$$

with $V_{1}(0)=0$ and $V_{1}(M)=1$.

Let $\tau$ be the first time the process $\left\{x^{m}\right\}$ reaches one of the absorbing states, i.e.

$$
\tau=\min \left\{m: x^{m}=0, M\right\} .
$$

Then $V_{1}(x)=\mathrm{P}\left(x^{\tau}=M\right)$ and it can be calculated, using Doob's optional stopping theorem (see [1]), that

$$
\mathrm{E}\left[x^{\tau} \mid x^{0}=x\right]=V_{1}(x) M+\left(1-V_{1}(x)\right) 0=x .
$$

Therefore, the expected return to player $I$ is

$$
V_{1}(x)=\frac{x}{M}
$$

A similar argument can be used to show that the expected return to player II is given by

$$
V_{2}(x)=\frac{M-x}{M}
$$

We note that, for every $x \in S$, the expected returns to the two players do not depend on the strategy profiles they use. Therefore, until time $\tau$, any pair of strategies with positive bets is a Nash equilibrium for this game.

Remark 3.1. Since the state space $S$ is discrete, the players are not allowed to bid 0 if they have a positive fortune and the process of player $I$ fortunes is a supermartingale; this process must then converge to 0 or $M$ with probability 1 . Hence, the stopping time $\tau$ defined in (3.1) is finite with probability 1 .

For a subfair game for player $I$, that is, for $0<w<1$, we can prove the following result.

Theorem 3.2. In a weighted two-person red-and-black game that is subfair to player I, i.e. $0<w<1$, a Nash equilibrium is for player I to play a bold strategy, i.e. $a(x)=x$ for $x=1, \ldots, M-1$, and for player II to play a timid strategy, i.e. $b(x)=1, x=1, \ldots, M-1$.

Proof. Set $Q(x)=\mathrm{P}_{x}$ [player $I$ reaches $\left.M\right]$, that is, the probability for player $I$ to reach $M$, starting with a fortune $x$, when player $I$ adopts a bold strategy $(a(x)=x$ for $x=1, \ldots, M-1)$ and player $I I$ uses a timid strategy $(b(x)=1$ for $x=1, \ldots, M-1)$. The corresponding law of motion for player $I$ at stage $m$ of the game is given by

$$
x^{m+1}= \begin{cases}x^{m}+1 & \text { with probability } \frac{x^{m}}{x^{m}+1} w, \\ 0 & \text { with probability } 1-\frac{x^{m}}{x^{m}+1} w .\end{cases}
$$


Considering $Q(\cdot)$ for each possible value of $x$, we get the following system of equations:

$$
\begin{aligned}
Q(1) & =w \frac{1}{2} Q(2), \\
Q(2) & =w \frac{2}{3} Q(3), \\
\vdots \quad & \vdots \quad \vdots \\
Q(M-2) & =w \frac{M-2}{M-1} Q(M-1), \\
Q(M-1) & =w \frac{M-1}{M} Q(M)=w \frac{M-1}{M} .
\end{aligned}
$$

Solving the system, we obtain the following general expression for $Q(x)$ :

$$
Q(x)=w^{M-x} \frac{x}{M} \quad \text { for } x=1, \ldots, M-1 .
$$

In order to prove that for player $I$ a bold strategy is optimal when player $I I$ plays timidly, it suffices to show that $Q(\cdot)$ is excessive (see Theorem 3.10 in Maitra and Sudderth [4]) or, equivalently, that the following inequality holds:

$$
w \frac{a}{a+1} Q(x+1)+\left(1-w \frac{a}{a+1}\right) Q(x-a) \leq Q(x)
$$

for every $x \in S$ and for every $a \in\{0,1, \ldots, x\}$.

With this inequality we are saying that, assuming player $I I$ adopts a timid strategy, the expected return player $I$ gets by initially staking an amount $1 \leq a \leq x$, and then playing boldly for the rest of the game, is always less than or equal to the expected return he gets by playing a bold strategy from the beginning. Note that, assuming player $I I$ plays timidly and player $I$ bids an amount $1 \leq a \leq x$, the law of motion for player $I$ at the first stage of the game is given by

$$
x^{1}= \begin{cases}x+1 & \text { with probability } \frac{a}{a+1} w, \\ x-a & \text { with probability } 1-\frac{a}{a+1} w .\end{cases}
$$

We now prove that (3.3) holds for all $x \in\{1, \ldots, M-1\}$, all $a \in\{1, \ldots, x\}$, and $0<w<1$. We substitute the expression for $Q(\cdot)$ given in (3.2) into (3.3), and we find that

$$
w \frac{a}{a+1} w^{M-x-1} \frac{x+1}{M}+\left(1-w \frac{a}{a+1}\right) w^{M-x+a} \frac{x-a}{M} \leq w^{M-x} \frac{x}{M} .
$$

Since $M$ is positive, we may multiply every term of the inequality by $M$, and divide by $w^{M-x}$ to get

$$
\frac{a}{a+1}(x+1)+\left(1-w \frac{a}{a+1}\right) w^{a}(x-a) \leq x .
$$

Next, we multiply every term by $(a+1)$ :

$$
a(x+1)+(a+1-w a) w^{a}(x-a) \leq x(a+1) .
$$

This is equivalent to

$$
a(x+1)+w^{a}(a+1)(x-a)-a w^{a+1}(x-a) \leq x(a+1),
$$


which simplifies to

$$
(x-a)\left(w^{a}(a+1)-a w^{a+1}\right) \leq x-a .
$$

This inequality clearly holds if $a=x$. For $a<x$ it reduces to

$$
w^{a}(a+1)-a w^{a+1} \leq 1 .
$$

We can show that $f(w)=w^{a}(a+1-a w)$ is an increasing function in $w$ and so achieves its maximum at $w=1$. Indeed,

$$
\frac{\mathrm{d} f(w)}{\mathrm{d} w}=a(a+1) w^{a-1}-a(a+1) w^{a}=a(a+1) w^{a-1}\left(1-w^{a}\right)>0 \quad \text { for } 0<w<1 .
$$

In conclusion, the inequality (3.3) holds for all $x$ and $0<w<1$, and, therefore, $Q(\cdot)$ is excessive for player $I$.

Now set $T(x)=\mathrm{P}_{M-x}$ [player $I I$ reaches $M$ ], which is the probability for player $I I$ to reach $M$ when he has an initial fortune of $M-x$ units and plays timidly, while player $I$ plays boldly. In this class of games, one player reaches the goal and the other reaches 0 with probability 1 ; hence,

$$
T(x)=1-Q(x)=1-w^{M-x} \frac{x}{M} .
$$

In order to prove that the timid strategy is optimal for player $I I$ when player $I$ plays boldly, as before it suffices to show that $T(\cdot)$ is excessive. So we show, assuming that player $I$ adopts a bold strategy, that the expected return player II gets by staking an amount $1 \leq b \leq M-x$ at the first stage of the game and then playing timidly is always less than or equal to the expected return he gets from playing timidly from the beginning.

Assuming that player $I$ plays boldly, but that player $I I$ bids an amount $1 \leq b \leq M-x$, the law of motion for player $I$ at the first stage of the game is

$$
x^{1}= \begin{cases}x+b & \text { with probability } \frac{x}{x+b} w \\ 0 & \text { with probability } 1-\frac{x}{x+b} w .\end{cases}
$$

Therefore, we have to prove that the following inequality holds for all $1 \leq b \leq M-x$ and all $0<w<1$ :

$$
w \frac{x}{x+b} T(x+b)+\left(1-w \frac{x}{x+b}\right) T(0) \leq T(x) .
$$

From (3.5) we get the expression for the function $T(\cdot)$. Therefore,

$$
w \frac{x}{x+b}\left(1-w^{M-x-b} \frac{x+b}{M}\right)+\left(1-w \frac{x}{x+b}\right) \leq 1-w^{M-x} \frac{x}{M},
$$

which reduces to

$$
1-\frac{x}{M} w^{M-x-b+1} \leq 1-w^{M-x} \frac{x}{M} .
$$

This is equivalent to $1 \leq w^{1-b}$. Hence, inequality (3.6) is verified, and the timid strategy is optimal for player $I I$, assuming that player $I$ uses a bold strategy.

Corollary 3.1. The profile (bold, timid), where the first description corresponds to the strategy of player I and the second to that of player II, is the unique Nash equilibrium for a weighted two-person red-and-black game that is subfair to player I. 
Proof. First, we show that if player II plays timidly, then 'bold' is the unique optimal strategy for player $I$. In fact, the only strategy for which (3.4) is actually an equality is the bold strategy; for any other strategy we have a strict inequality.

In the same way, we show that if player $I$ plays boldly, the 'timid' strategy is the unique optimal strategy for player $I I$, since that is the only strategy for which (3.7) is an equality.

From Lemma A.1 in Appendix A, below, it follows that the profile (bold, timid) is the unique Nash equilibrium for this game.

Remark 3.2. The formulation of this game comes from the idea that $w$ can be considered to be a penalizing weight imposed on one of the players. For example, we can imagine a situation in which there is a state agency that has the power to control speculators by penalizing their probabilities of winning. The result given in Theorem 3.2 gives a Nash equilibrium when the penalizing weight is imposed on player $I$, making the game subfair to him.

\section{Proportional two-person red-and-black}

Suppose that, at stage $m$, player $I$ has $1 \leq x^{m} \leq M-1$ units of money and he bids an amount $1 \leq a \leq x^{m}$, while player $I I$ bids $1 \leq b \leq M-x^{m}$. In a proportional two-person red-and-black game the law of motion for player $I$ at stage $m$ is defined by

$$
x^{m+1}= \begin{cases}x^{m}+b & \text { with probability } \frac{w a}{w a+\bar{w} b}, \\ x^{m}-a & \text { with probability } \frac{\bar{w} b}{w a+\bar{w} b},\end{cases}
$$

where $w$ and $\bar{w}=1-w$ are constant values between 0 and 1 .

Remark 4.1. For $w=\frac{1}{2}$, we have a fair, weighted two-person red-and-black game, for which we proved, in Theorem 3.1, that any pair of strategies with positive bets is a Nash equilibrium.

Remark 4.2. For every strategy profile, the process $\left\{x^{m}\right\}$ of player $I$ fortunes is a supermartingale if $w \leq \frac{1}{2}$ and a submartingale if $w \geq \frac{1}{2}$.

Let us now consider the more interesting case of $w \neq \frac{1}{2}$.

Theorem 4.1. In a proportional two-person red-and-black game that is subfair to player I, i.e. $0<w<\frac{1}{2}$, it is Nash for player I to play a bold strategy, i.e. $a(x)=x$ for $x=1, \ldots, M-1$ and for player II to play a timid strategy, i.e. $b(x)=1$ for $x=1, \ldots, M-1$. Therefore, the profile (bold, timid) is a Nash equilibrium for this class of games. If the game is superfair to player I and subfair to player II, then the profile (timid, bold) is a Nash equilibrium.

Proof. Set $Q(x)=\mathrm{P}_{x}$ [player $I$ reaches $\left.M\right]$, that is, the probability for player $I$ to reach his goal $M$, starting at $x$, supposing that player $I$ plays a bold strategy and player $I I$ plays a timid strategy. The corresponding law of motion for player $I$ at stage $m$ is

$$
x^{m+1}= \begin{cases}x^{m}+1 & \text { with probability } \frac{w x^{m}}{w x^{m}+\bar{w}}, \\ 0 & \text { with probability } \frac{\bar{w}}{w x^{m}+\bar{w}} .\end{cases}
$$


Considering the values of $Q(\cdot)$ for all possible $x$, we obtain the following system of equations:

$$
\begin{aligned}
Q(1) & =w Q(2), \\
Q(2) & =w \frac{2}{w+1} Q(3), \\
\vdots \quad & \vdots \quad \\
Q(M-2) & =w \frac{M-2}{w(M-3)+1} Q(M-1), \\
Q(M-1) & =w \frac{M-1}{w(M-2)+1} .
\end{aligned}
$$

Solving the system, we find, for $x=1, \ldots, M-1$, the following general expression for $Q(x)$ :

$$
Q(x)=w^{M-x} \prod_{i=x}^{M-1}\left(\frac{i}{w(i-1)+1}\right) .
$$

In order to prove that the bold strategy is optimal for player $I$, we show that $Q(\cdot)$ is excessive.

Assuming that player $I I$ plays a timid strategy and player $I$ stakes an amount $1 \leq a \leq x$ at the first stage and then plays boldly, we find the following law of motion for player $I$ at the first stage of the game:

$$
x^{1}= \begin{cases}x+1 & \text { with probability } \frac{w a}{w a+\bar{w}}, \\ x-a & \text { with probability } \frac{\bar{w}}{w a+\bar{w}} .\end{cases}
$$

We want to show that, for $0<w<\frac{1}{2}$, the following inequality holds:

$$
\frac{w a}{w a+\bar{w}} Q(x+1)+\frac{\bar{w}}{w a+\bar{w}} Q(x-a) \leq Q(x) .
$$

From (4.1) we obtain the expression for the function $Q(\cdot)$ and, therefore, the desired inequality (4.2) becomes

$$
\begin{aligned}
& \frac{w a}{w a+\bar{w}} w^{M-(x+1)} \prod_{i=x+1}^{M-1}\left(\frac{i}{w(i-1)+1}\right)+\frac{\bar{w}}{w a+\bar{w}} w^{M-(x-a)} \prod_{i=x-a}^{M-1}\left(\frac{i}{w(i-1)+1}\right) \\
& \quad \leq w^{M-x} \prod_{i=x}^{M-1}\left(\frac{i}{w(i-1)+1}\right) .
\end{aligned}
$$

Multiplying both sides by the positive quantity

$$
(w(a-1)+1)\left[w^{M-x} \prod_{i=x}^{M-1} \frac{i}{w(i-1)+1}\right]^{-1}
$$

gives

$$
a\left(\frac{w(x-1)+1}{x}\right)+(1-w) w^{a} \prod_{i=x-a}^{x-1}\left(\frac{i}{w(i-1)+1}\right) \leq w(a-1)+1
$$


and, by taking the first term on the left-hand side to the right-hand side, we find that

$$
(1-w) w^{a} \prod_{i=x-a}^{x-1}\left(\frac{i}{w(i-1)+1}\right) \leq w(a-1)+1-a\left(\frac{w(x-1)+1}{x}\right) .
$$

Using elementary algebra we then obtain

$$
(1-w) w^{a} \prod_{i=x-a}^{x-1}\left(\frac{i}{w(i-1)+1}\right) \leq \frac{(x-a)(1-w)}{x} .
$$

This is equivalent to

$$
w^{a} \prod_{i=x-a}^{x-1}\left(\frac{i}{w(i-1)+1}\right) \leq \frac{x-a}{x} .
$$

Notice that, for $a=1,2, \ldots, x-1$, we have

$$
w \frac{x-a}{w(x-a-1)+1}<\frac{x-a}{x-a+1},
$$

since $w(x-a+1)<w(x-a-1)+1$ because $w<\frac{1}{2}$.

So, rewriting the inequality (4.3) extensively, we have

$$
\begin{gathered}
w \frac{x-1}{w(x-2)+1} w \frac{x-2}{w(x-3)+1} \cdots w \frac{x-a}{w(x-a-1)+1} \\
\quad \leq \frac{x-1}{x} \frac{x-2}{x-1} \cdots \frac{x-a}{x-a+1}=\frac{x-a}{x} .
\end{gathered}
$$

This completes the proof of the inequality (4.3).

We now have to show that the timid strategy is optimal for player $I I$ when player $I$ plays a bold strategy. Let $T(x)=\mathrm{P}_{M-x}$ [player $I I$ reaches $M$ ], the probability that player $I I$ reaches $M$ when he has $M-x$ units of money and plays a timid strategy, while player $I$ plays a bold strategy. A general formula for $T(x), x=1, \ldots, M-1$, is given by

$$
T(x)=1-Q(x)=1-w^{M-x} \prod_{i=x}^{M-1}\left(\frac{i}{w(i-1)+1}\right) .
$$

We consider the case in which player II first plays $1 \leq b \leq M-x$ and then plays a timid strategy, and show that $T(\cdot)$ is excessive when player $I$ plays a bold strategy. The law of motion for player $I$ at the first stage of the game is given by

$$
x^{1}= \begin{cases}x+b & \text { with probability } \frac{w a}{w a+\bar{w} b}, \\ 0 & \text { with probability } \frac{\bar{w} b}{w a+\bar{w} b} .\end{cases}
$$

We need to show that, for $w \leq \frac{1}{2}$, the following inequality holds:

$$
\frac{w x}{w x+\bar{w} b} T(x+b)+\frac{\bar{w} b}{w x+\bar{w} b} T(0) \leq T(x) .
$$


Using (4.4), we can rewrite this inequality as

$$
\begin{gathered}
\frac{w x}{w x+\bar{w} b}\left(1-w^{M-x-b} \prod_{i=x+b}^{M-1}\left(\frac{i}{w(i-1)+1}\right)\right)+\frac{\bar{w} b}{w x+\bar{w} b} \\
\leq\left(1-w^{M-x} \prod_{i=x}^{M-1}\left(\frac{i}{w(i-1)+1}\right)\right) .
\end{gathered}
$$

Since $w x /(w x+\bar{w} b)+\bar{w} b /(w x+\bar{w} b)=1$, we have

$$
1-\frac{w x}{w x+\bar{w} b} w^{M-x-b} \prod_{i=x+b}^{M-1}\left(\frac{i}{w(i-1)+1}\right) \leq 1-w^{M-x} \prod_{i=x}^{M-1}\left(\frac{i}{w(i-1)+1}\right) .
$$

This is equivalent to

$$
\frac{x}{w x+\bar{w} b} w^{M-x-b+1} \prod_{i=x+b}^{M-1}\left(\frac{i}{w(i-1)+1}\right) \geq w^{M-x} \prod_{i=x}^{M-1}\left(\frac{i}{w(i-1)+1}\right) .
$$

Since $x /(w x+\bar{w} b)=x /[b+w(x-b)]$, dividing both sides by

$$
w^{M-x-b+1} \prod_{i=x+b}^{M-1} \frac{i}{w(i-1)+1}
$$

gives

$$
w^{b-1} \prod_{i=x}^{x+b-1}\left(\frac{i}{w(i-1)+1}\right) \leq \frac{x}{b+w(x-b)}
$$

which we prove by induction.

We start by showing that the inequality holds for $b=2$, since for $b=1$ we have the exact equality. So, for $b=2$ and for $x=1, \ldots, M-1$, we have

$$
w \frac{x}{w(x-1)+1} \frac{x+1}{w x+1} \leq \frac{x}{2+w(x-2)} .
$$

This is equivalent to

$$
(2+w x-2 w)(w x+w) \leq(w x-w+1)(w x+1),
$$

which holds if and only if

$$
2 w^{2}-3 w+1 \geq 0
$$

and, in turn, this inequality is satisfied for $w \leq \frac{1}{2}$.

We assume that inequality (4.5) holds for $b=n \geq 2$; this means that, for $w \leq \frac{1}{2}$, we have

$$
w^{n-1} \prod_{i=x}^{x+n-1}\left(\frac{i}{w(i-1)+1}\right) \leq \frac{x}{n+w(x-n)} .
$$


To prove that the inequality holds for $b=n+1$, first notice that

$$
w^{n} \prod_{i=x}^{x+n}\left(\frac{i}{w(i-1)+1}\right)=w^{n-1} \prod_{i=x}^{x+n-1}\left(\frac{i}{w(i-1)+1} w \frac{x+n}{w(x+n-1)+1}\right) .
$$

By (4.6) we then obtain

$$
w^{n} \prod_{i=x}^{x+n}\left(\frac{i}{w(i-1)+1}\right) \leq \frac{x}{n+w(x-n)} w \frac{x+n}{1+w(x+n-1)} .
$$

It now suffices to show that, for $w \leq \frac{1}{2}$,

$$
\frac{x}{n+w(x-n)} w \frac{x+n}{1+w(x+n-1)} \leq \frac{x}{n+1+w(x-n-1)} .
$$

This is equivalent to

$$
(w x+w n)(n+1+w x-w n-w) \leq(n+w x-w n)(w x+w n-w+1),
$$

which simplifies to

$$
2 w^{2}-3 w+1 \geq 0
$$

and this is satisfied for $w \leq \frac{1}{2}$. (Note that we get the same quadratic function of $w$ for any value we chose for $b$ ).

Therefore, we can conclude that $T(\cdot)$ is excessive for player II and that the profile (bold, timid) is a Nash equilibrium for this class of games. Using symmetry, we can show that if the game is superfair to player $I$ and subfair to player $I I$, then (timid, bold) is a Nash equilibrium.

Remark 4.3. Dubins and Savage [3] showed, for the original version of the red-and-black game, that there are other optimal strategies (in addition to bold play) for the subfair case, i.e. $w<\frac{1}{2}$. In contrast, there is a unique Nash equilibrium for our games.

Corollary 4.1. The profile (bold, timid) is the unique Nash equilibrium for a proportional twoperson red-and-black game that is subfair to player I. Also, the profile (timid, bold) is the unique Nash equilibrium for a proportional two-person red-and-black game that is superfair to player $I$.

Proof. Consider a proportional two-person red-and-black game that is subfair to player $I$. We can show that if player II plays timidly, then bold is the unique optimal strategy for player $I$. In fact, the only strategy for which (4.2) is actually an equality is the bold strategy; for any other strategy we have a strict inequality.

In the same way, we can show that if player I plays boldly, the timid strategy is the unique optimal strategy for player $I I$, since that is the only strategy for which (4.7) is an equality.

In the subfair version of the game, we can show that if player $I$ plays boldly, then timid is the unique optimal strategy for player $I I$, and vice versa.

The uniqueness of these Nash equilibria follows from Lemma A.1 in Appendix A. 


\section{Proportional $N$-person red-and-black}

We consider a formulation of the proportional red-and-black game with $N$ players, denoted by $1,2, \ldots, N$. At each stage $m$, each player has an amount of money $x_{j}^{m}, j=1, \ldots N$, and wants to reach the target $M$, that is, the fixed total amount of money in the system (i.e. $\sum_{j=1}^{N} x_{j}^{m}=M$ for all $m$ ).

The state space is given by the set of all possible $N$-tuples of the fortunes of the $N$ players. At each stage, each player bids an amount of money $a_{j}^{m} \in\left\{1, \ldots, x_{j}^{m}\right\}$ if $1 \leq x_{j}^{m} \leq M-1$, and $a_{j}^{m}=0$ otherwise, for $j=1, \ldots, N$. The probability of each player winning the bet is proportional to his weighted bet.

During the game, one or more players can go broke, which means that they are out of the game. Hence, let $k^{m}$ be the number of players active in the game at stage $m$ and, for each player $j$, define the corresponding weight

$$
w_{j}^{m}= \begin{cases}0 & \text { if } x_{j}^{m}=0, \\ \frac{w_{j}^{m-1}}{\sum_{\left\{i: x_{i}^{m}>0\right\}} w_{i}^{m-1}} & \text { if } x_{j}^{m}>0,\end{cases}
$$

such that $0 \leq w_{j}^{m}<1$ and $\sum_{j=1}^{N} w_{j}^{m}=1$ for all $m$. In fact, every time a player has to leave the game, the value of the weights associated with the remaining (active) players must be redefined, so that they still sum to one.

The law of motion for player $j$ at stage $m$ is

$$
x_{j}^{m+1}= \begin{cases}x_{j}^{m}-a_{j}^{m}+\sum_{i=1}^{N} a_{i}^{m} & \text { with probability } \frac{w_{j}^{m} a_{j}^{m}}{\sum_{i=1}^{N} w_{i}^{m} a_{i}^{m}}, \\ x_{j}^{m}-a_{j}^{m} & \text { with probability } 1-\frac{w_{j}^{m} a_{j}^{m}}{\sum_{i=1}^{N} w_{i}^{m} a_{i}^{m}} .\end{cases}
$$

Assuming that $w_{i}^{m}=1 / k^{m}$ for all $m$ and all $i$ such that $x_{i}^{m}>0$, we have a fair game for all the players, in terms of the definition given in Section 3.

Theorem 5.1. In a fair, proportional $N$-person red-and-black game, any profile of strategies with positive bets for each player $j$, up to the time that $x_{j}^{m}$ reaches 0 or $M$, is Nash.

Proof. The proof is omitted since it is analogous to the proof of Theorem 3.1.

When the game is not fair to all the players, i.e. when at some stage $t$ there are at least two players with a value for their weights $w_{j}^{t}$ not exactly equal to $1 / k^{t}$, we would like to extend to the $N$-person model the results we found for proportional two-person red-and-black.

Our conjecture is that, for any player $j$ to whom the game is subfair, it is Nash to play a bold strategy and, for any player $i$ to whom the game is superfair, it is Nash to play a timid strategy.

First, we need to specify what we mean by subfair and superfair in this $N$-person version of the game. We could say that at some stage $t$ the proportional $N$-person red-and-black game is subfair to player $j$ when $0<w_{j}^{t}<1 / k^{t}$ and superfair to player $i$ when $1 / k^{t}<w_{i}^{m}<1$. During the game, the scenario for the single player can change, since it could happen that the game is subfair to player $j$ until all the players are active, but that, after one of them is out of the game, the game becomes superfair to him. For this reason, it may be optimal for a player to start with one strategy and then switch to a different one during the game. So, in order to 
give a definition of subfair and superfair games and then establish the Nash strategies for the $N$ players, it seems very important to consider the number $k^{m}$ of players who are active in the game at each stage $m$. Some results about the original version of the red-and-black game with $k$ players can be found in Pendergrass and Siegrist [5].

In conclusion, we make the following conjecture.

Conjecture 5.1. Given the number $k^{m}$ of players active at stage $m$ of the game, with $2 \leq k^{m} \leq$ $N$, it is Nash for a player $j$ to use a bold strategy if the current weight $w_{j}^{m}$ associated with him is less than or equal to $1 / k^{m}$, and it is Nash for him to use a timid strategy otherwise.

Examples in which the conjecture is verified can be found in Pontiggia [6].

Remark 5.1. Even for an $N$-person red-and-black game with constant probabilities of winning, it is hard to extend the trivial result given in Remark 2.1 for the two-person version. In this game we found that, contrary to intuition, the function that represents the return to one of the players when all of them adopt a timid strategy is not a monotone function with respect to his fortune. So, at some stage of the game it may be advantageous for the player to give one of his opponents a unit of money. For this reason, a coupling argument to prove our conjecture that the timid profile is a Nash equilibrium for this game does not work. Several examples involving this class of games are given in Pontiggia [6].

\section{Appendix A.}

The games considered in this paper can be treated as zero-sum games, since the games end with probability 1 .

As suggested by Andrew McLennan (private communication), the general result given in the following lemma can be applied to our games. Even if it seems very easy to prove and very intuitive, the author could not find any reference to this lemma in the literature.

Lemma A.1. Assume that, in a two-person zero-sum game, $\left(\mu^{*}, v^{*}\right)$ is a Nash equilibrium, $\mu^{*}$ is the unique optimal strategy for player I against $v^{*}$, and $v^{*}$ is the unique optimal strategy for player II against $\mu^{*}$. Then, $\left(\mu^{*}, v^{*}\right)$ is the unique Nash equilibrium for the game.

Proof. Let $\psi_{1}$ denote the payoff to player $I$ and let $\mathrm{E}_{\mu, \nu} \psi_{1}$ be the expected payoff to $I$ from the profile $(\mu, v)$. It is known that if $\left(\mu^{*}, \nu^{*}\right)$ is a Nash equilibrium, then $\mathrm{E}_{\mu^{*}, v^{*}} \psi_{1}$ is the value of the game, denoted by $V$.

Suppose now that we have another Nash equilibrium for the game, given by the strategy profile $(\tilde{\mu}, \tilde{v})$. The fact that $\left(\mu^{*}, v^{*}\right)$ and $(\tilde{\mu}, \tilde{v})$ are Nash equilibria implies that

$$
\begin{gathered}
\mathrm{E}_{\mu^{*}, \nu} \psi_{1} \geq \mathrm{E}_{\mu^{*}, v^{*}} \psi_{1} \geq \mathrm{E}_{\mu, \nu^{*}} \psi_{1} \quad \text { for all } \mu, v, \\
\mathrm{E}_{\tilde{\mu}, \nu} \psi_{1} \geq \mathrm{E}_{\tilde{\mu}, \tilde{\nu}} \psi_{1} \geq \mathrm{E}_{\tilde{\mu}, \nu} \psi_{1} \quad \text { for all } \mu, \nu .
\end{gathered}
$$

Therefore,

$$
\mathrm{E}_{\mu^{*}, \nu^{*}} \psi_{1} \leq \mathrm{E}_{\mu^{*}, \tilde{v}} \psi_{1} \leq \mathrm{E}_{\tilde{\mu}, \tilde{v}} \psi_{1} \leq \mathrm{E}_{\tilde{\mu}, \nu^{*}} \psi_{1} \leq \mathrm{E}_{\mu^{*}, v^{*}} \psi_{1} .
$$

Since the same quantity appears on both the extreme left- and right-hand sides, all the inequalities must be equalities, which proves that

$$
V=\mathrm{E}_{\tilde{\mu}, \tilde{v}} \psi_{1}=\mathrm{E}_{\mu^{*}, \nu^{*}} \psi_{1}=\mathrm{E}_{\tilde{\mu}, v^{*}} \psi_{1}=\mathrm{E}_{\mu^{*}, \tilde{v}} \psi_{1} .
$$

Moreover, the profile $\left(\mu^{*}, \tilde{v}\right)$ is also a Nash equilibrium, since

$$
\mathrm{E}_{\mu, \tilde{v}} \psi_{1} \leq \mathrm{E}_{\tilde{\mu}, \tilde{v}} \psi_{1}=\mathrm{E}_{\mu^{*}, \tilde{v}} \psi_{1}=\mathrm{E}_{\mu^{*}, v^{*}} \psi_{1} \leq \mathrm{E}_{\mu^{*}, \nu} \psi_{1} .
$$


Hence, $\tilde{v}$ is optimal against $\mu^{*}$ and, therefore, $\tilde{v}=v^{*}$. Using the same procedure, we can show that $\left(\tilde{\mu}, v^{*}\right)$ is also a Nash equilibrium. Hence, $\tilde{\mu}$ is optimal against $\nu^{*}$ and, therefore, $\tilde{\mu}=\mu^{*}$.

\section{Acknowledgements}

This article is part of my doctoral thesis at the University of Minnesota. I wish to thank my advisor William Sudderth for his suggestions and guidance. I am greatly indebted to Andrew McLennan for providing me with essential suggestions for proving the uniqueness of the Nash equilibria found in Theorems 3.2 and 4.1. Finally, I would like to thank the referee for his very helpful comments and feedback.

\section{References}

[1] Doob, J. L. (1953). Stochastic Processes. John Wiley, New York.

[2] Dubins, L. (1998). Discrete red-and-black with fortune-dependent win probabilities. Prob. Eng. Inf. Sci. 12, 417-424.

[3] Dubins, L. E. And Savage, L. J. (1976). Inequalities for Stochastic Processes: How to Gamble if You Must, 2nd edn. Dover, New York.

[4] Maitra, A. P. And Sudderth, W. D. (1996). Discrete Gambling and Stochastic Games. Springer, New York.

[5] Pendergrass, M. And Siegrist, K. (2001). Generalizations of bold play in red and black. Stoch. Process Appl. 92, 163-180.

[6] Pontiggia, L. (2004). Topics in stochastic games. Doctoral Thesis, University of Minnesota, Minneapolis.

[7] Ross, S. M. (1974). Dynamic programming and gambling models. Adv. Appl. Prob. 6, 598-606.

[8] SECCHI, P. (1997). Two-person red-and-black stochastic games. J. Appl. Prob. 34, 107-126. 